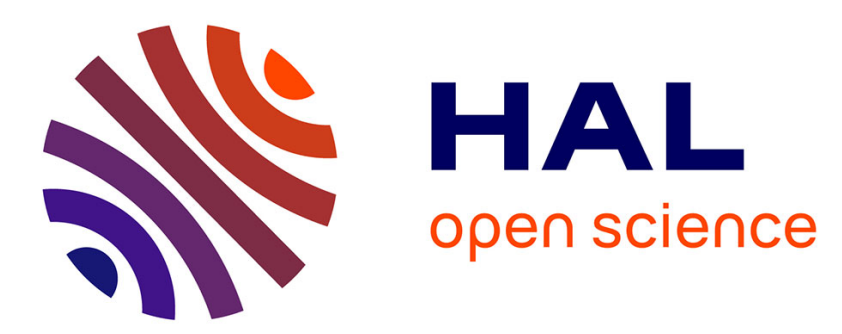

\title{
Optical Power Control to Efficiently Handle Flex-Grid Spectrum Gain over Existing Fixed-Grid Network Infrastructures
}

Mohamad Matthieu Kanj, Esther Le Rouzic, Djamel Amar, Jean-Luc Auge, Bernard Cousin, Nicolas Brochier

\section{To cite this version:}

Mohamad Matthieu Kanj, Esther Le Rouzic, Djamel Amar, Jean-Luc Auge, Bernard Cousin, et al.. Optical Power Control to Efficiently Handle Flex-Grid Spectrum Gain over Existing Fixed-Grid Network Infrastructures. International Conference on Computing, Networking and Communications (ICNC 2016), Feb 2016, Kauai, United States. 10.1109/ICCNC.2016.7440674 · hal-01216701

\author{
HAL Id: hal-01216701 \\ https://hal.science/hal-01216701
}

Submitted on 16 Oct 2015

HAL is a multi-disciplinary open access archive for the deposit and dissemination of scientific research documents, whether they are published or not. The documents may come from teaching and research institutions in France or abroad, or from public or private research centers.
L'archive ouverte pluridisciplinaire HAL, est destinée au dépôt et à la diffusion de documents scientifiques de niveau recherche, publiés ou non, émanant des établissements d'enseignement et de recherche français ou étrangers, des laboratoires publics ou privés. 


\title{
Optical Power Control to Efficiently Handle Flex-Grid Spectrum Gain over Existing Fixed-Grid Network Infrastructures
}

\author{
M. Kanj ${ }^{1}$, E. Le Rouzic ${ }^{2}$, D. Amar ${ }^{2}$, J.L. Auge 2 , B. Cousin ${ }^{1}$, N. Brochier ${ }^{2}$ \\ ${ }^{1} \mathrm{~b}<>$ com, 1219 avenue Champs Blancs, 35510 Cesson-Sévigné, France (Mohamad.KANJ@b-com.com). \\ 2 Orange Labs, Lannion, France.
}

\begin{abstract}
The exponential traffic growth in optical networks has triggered the evolution from Fixed-Grid to Flex-Grid technology. This evolution allows better spectral efficiency and spectrum usage over current networks in order to facilitate dynamic and huge traffic demands. The integration of Flex-Grid technology increases the number of optical channels established over optical links, leading, however, to an increase in amplification power and possibly saturating optical amplifiers.

In this work, we propose a power adaptation process that takes advantage of link optical signal to noise ratio (OSNR) margins to allow network operators to support this power increase while maintaining the use of legacy amplifiers. Results show that controlling channel optical power benefits from the Flex-Grid in terms of spectrum and capacity gain using in-place amplifier infrastructure.

Index Terms-GMPLS, Link Design, Flex-Grid, Power Con-
\end{abstract} trol, Path Computation Algorithm.

\section{INTRODUCTION}

Internet services (video conferencing, cloud services and video streaming) and consequently traffic demands are increasing continually, leading to huge traffic growth in the core optical network. There is a need for network operators to increase their optical network capacity to follow this traffic growth. Since the deployment of new optical fibers is still very expensive, network operators are pushing to exploit the totality of their network capacity by optimizing their optical resources, and thus postponing the deployment of new infrastructures. This exploitation requires new technologies and flexible equipment that are able to handle different types of optical channels, from small to extremely high data rates [1].

Fixed-Grid technology is no longer qualified to handle the increasing data rates of optical channels. At the same time, the $50 \mathrm{GHz}$ ITU grid, due to its fixed-spectrum spacing, produces losses of spectrum resources when the bandwidth occupancy of the established demands is smaller than (or is not an exact multiple of) the size of the allocated spectrum slots [2].

The ITU recommendation G.694.1 [3] for a Flex-Grid optical network has defined a new flexible spectral grid standard for wavelength division multiplexing (WDM) applications. This flexible spectral grid has a smaller slot granularity of $12.5 \mathrm{GHz}$, with nominal central frequency on a grid of 6.25 $\mathrm{GHz}$ spacing compared to the currently $50 \mathrm{GHz}$ Fixed-Grid.
This recommendation has made the Flex-Grid a promising technology that is capable of following traffic growth and various traffic demands. Flex-Grid efficiently uses available spectrum resources, especially when associated with novel coherent transmission technologies and advanced modulation formats.

Switching from Fixed-Grid to Flex-Grid technology has an impact on the optical amplifiers of an already deployed optical network. Indeed, the optical amplifiers present at the end of each successive span constituting an optical link and in switching nodes are designed and engineered for a FixedGrid WDM network. Since Flex-Grid technology allows the reduction of channel spacing, it allows the possibility to create new optical channels over the saved spectrum. However, this increase in the number of optical channels increases the optical power injected in optical links and may cause unwanted impairments due to the saturation of some amplifiers in the already deployed network.

In the literature, several studies have focused on developing accurate physical impairment estimators over uncompensated links. They have demonstrated the existence of an optimal optical power channel that leads to minimum impairment generation and thus achieves better transmission performance (maximum reach) [4] [5] [6] [7]. Others have focused on improving link performances (achieving SNR margin gain) and thus increasing network throughput by adapting channel launch power and optimizing spectral resources and modulation formats without taking into account power resource limits over optical links [8] [9].

In this paper, unlike the current paradigm that aims to optimize channel power to their optimum values regardless their reaches, we propose to control and adapt the power of these channels to their minimum required performances (adaptation to the real physical reach). This enables optical power margins to be used for overcoming the power limitation of amplifiers when increasing the number of channels over network links. For this purpose, we propose a distributed generalized multi-protocol label switching (GMPLS)-based control plane with resource reservation protocol-traffic engineering (RSVPTE) and open shortest path first-traffic engineering (OSFPTE) protocol modification that implements this power control process. Performance of the novel scheme is demonstrated 
with simulations.

This paper is organized as follows. Section II presents an overview of optical network design issues. Section III introduces the link power margin and our design method. Section IV presents the power control process. Section V presents GMPLS protocol modifications made to implement the power control process and its associated path computation algorithm. Section VI presents simulated scenarios and results. Conclusion and future works are presented in Section VII.

\section{OPTICAL LINK DESIGN AND POWER LIMITATIONS}

We consider a set of successive optical spans constituting an optical link between two optical nodes (e.g., reconfigurable optical add-drop multiplexers; ROADMs) as shown in Fig. 1. The optical link design consists of choosing the set of optical amplifiers that can compensate for span losses and simultaneously support the optical power of the total number of channels planned for that link, while seeking maximum optical performance. The link design has the objective of maximizing OSNR, minimizing linear and non-linear effects. Complexity of the process arises in particular because of the contradictory objectives of amplifiers; they must compensate for link span losses, satisfy the aggregate optical power for all optical channels sharing the fiber, and simultaneously minimize the amount of generated noise.

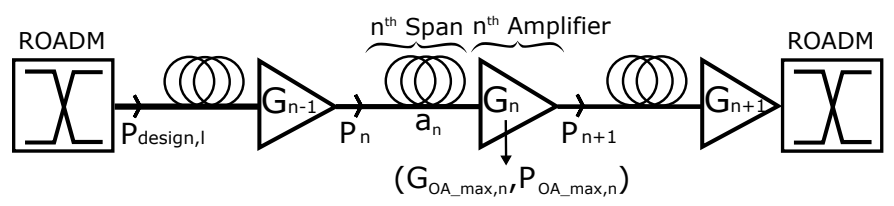

Fig. 1. Simplified representation of an amplified link (succession of a fiber span and optical amplifier) between two ROADMs.

As explained in the previous section, links in current FixedGrid WDM networks are designed to support a given number of channels $\left(N_{\text {channel_max }}\right)$. In general, every link $(l)$ has its own $N_{\text {channel_max, }}$; however, to ease our study, without any loss of generality, we assume that these numbers are identical all over the network. The use of Flex-Grid technology in these infrastructures may increase the number of channels in some links and thus their optical power level.

In fact, if the number of channels is not controlled and limited, there may be some risks of power saturation in the amplifiers that are already close to their maximum output power (power saturation limit) with the initial $N_{\text {channel_max }}$, leading to strong performance degradation on these links. Inversely, if the number of channels is limited to $N_{\text {channel_max }}$, the spectrum gain enabled by Flex-Grid technology cannot be exploited. Replacing optical amplifiers with new ones having bigger output power is a potential solution, but costly since it requires buying new amplifiers, interruption of the link, and full redesign. In this paper, we propose to make the information of optical power available to the control plane to benefit from Flex-Grid spectrum gain promises, while keeping the in-place amplifiers.
It is noteworthy that, at the end of design step, amplifiers are used in a fixed gain mode, which means that once the design step is finished, adjusted amplifier gains are never changed.

\section{LINK POWER MARGIN DEFINITION AND NOVEL DESIGN METHOD}

As a first step, we propose to control the optical power to benefit from the unused power left in each amplifier once the network is designed and deployed, which we call link power margin.

\section{A. Link Power Margin}

Let $N_{\text {channel_max }}$ be the maximum number of channels per each link. Let $P_{\text {design,l }}\left(P_{1}\right)$ be the input optical power designed for the link $l$ having $N_{\text {channel_max }}$. The difference between all span characteristics (losses, non-linearity coefficient, and length) leads to the use of different types of amplifiers with different characteristics in terms of maximum gain $\left(G_{O A \_\max }\right)$, maximum power $\left(P_{O A \_\max }\right)$, or noise figure $(N F)$ for each span. This variation results in having over every link $l$, different $P_{\text {design, } l}$ and thus $P_{\text {channel, } l}$ (individual channel power over link $l$ ). This $P_{\text {design, } l}$ when applied at link $l$ input, results in different span input powers depending on span attenuation and amplifier configured gains when going through the link. Therefore, there is a power margin ( $\left.P_{O A_{-} \text {margin, } n}\right)$ over the $n^{\text {th }}$ amplifier such that $P_{O A \_m a r g i n, n}=P_{O A \_m a x, n}-$ $P_{n+1}$, where $P_{n+1}$ is the power at the input of the $n+1^{t h}$ span.

We define as link power margin $P_{\text {margin, } l}$, the minimum power margin that exists over the amplifiers of link $l$. Therefore, the maximum optical power that can be applied at the input of link $l$ without saturation of any amplifier is $P_{\text {max }, l}=P_{\text {design }, l}+P_{\text {margin }, l}$. Our utilization of power margin complements recent works on design margins and system margins, as in [10] [11] [12]. In these works, the power control aspect was neglected. Here, we specifically focus on the control of the optical power. However, this requires fine knowledge of the maximum power allowed in each link, which in turn requires understanding of the link design step and the limitations of optical links.

\section{B. Design Method}

In order to evaluate our power control process, we have to precisely model the link design step. To this end, we have developed a link design method, which we briefly presented in [13], taking advantage of the optimization strategy presented in [14]. Note that our proposed power control can work with any other design method. The LOGON strategy proposed in [14] consists of performing a local optimization of the optical signal to noise ratio (OSNR) and non-linear impairments at span level, leading to a global OSNR optimization over all the links of the network. Therefore, it proposes to apply an optimal power spectral density at the input of every span, calculated using span and amplifier characteristics by applying formula (6) in [14]. 
Eq.(1) represents the aggregated optical power that corresponds to this power spectral density at the $n^{\text {th }}$ span input for $N_{\text {channel_max }}$ channels having $R_{s}$ spectrum width each, where $h, \mu, \bar{F}_{n}$, and $\rho_{N L I, n}$ stand for Planck's constant, the electromagnetic wave frequency, the NF of the $n^{\text {th }}$ amplifier, and non-linear effect contribution respectively.

$$
\begin{gathered}
P_{n}=\left(\frac{h \mu F_{n}}{2 \rho_{N L I, n}}\right)^{\frac{1}{3}} \\
F_{n}=F_{1, n}+\frac{F_{2, n} D_{n} G_{O A \_m a x, n}}{G_{n}^{2}} \\
G_{n}=a_{n} \frac{P_{n+1}}{P_{n}}
\end{gathered}
$$

Our link design method consists of choosing the optical amplifier that satisfies the link design constraints (maximize OSNR, minimize non-linear effect, satisfy $N_{\text {channel_max }}$ power, and compensate for span loss). Each amplifier NF is calculated using Eq. (2), and varies according to the adjusted gain $G_{n}$. We use variable gain dual-stage amplifiers without mid-stage access where $F_{1, n}$ and $F_{2, n}$ are the NF for the first and the second stage, respectively, and $D_{n}$ denotes the power ratio for both stages to account for the difference between preamp and booster performance. Eq. (3) calculates the desired amplifier gain $\left(G_{n}\right)$ to compensate for span loss, where $a_{n}$ is the attenuation of the $n^{\text {th }}$ span, $P_{n}$ is the power at the input of the $n^{t h}$ span, and $P_{n+1}$ is the power at the output of the $G_{n}$ optical amplifier as shown in Fig.1.

We replace $F_{n}$ in Eq. (2) by its value in Eq. (1), and then the $P_{n}$ in Eq. (3) by its value of Eq.(1) to attain a third degree polynomial equation represented by Eq. (4), which we solved analytically. The solution of this equation in Eq. (5) gives us the value of the gain that should be adjusted in the $n^{\text {th }}$ amplifier in order to obtain minimum linear and non-linear impairment generation.

$h \mu F_{1, n} G_{n}^{3}+h \mu F_{2, n} D_{n} G_{O A \_m a x, n} G_{n}-2 \rho_{N L I, n} a_{n}^{3} P_{n+1}^{3}=0$

$$
\begin{aligned}
& G_{n}=\sqrt{\frac{4 F_{2, n} D_{n} G_{O A \_} \max , n}{3 F_{1, n}}} \times \\
& \sinh \left(\frac{1}{3} \operatorname{asinh}\left(\frac{\rho_{N L I, n}\left(a_{n} P_{n+1}\right)^{3} \sqrt{\frac{27 F_{1, n}}{\left(F_{2, n} D_{n} G_{O A_{-} \max , n}\right)^{3}}}}{h \mu}\right)\right)
\end{aligned}
$$

Our link design is performed from the last span to the first one; the amplifier that can satisfy both required gain $\left(G_{n}\right)$ and optimum power $\left(P_{n+1}\right)$, while achieving the smallest NF is selected. If none of the amplifiers can satisfy these requirements, the one with the closest maximum power $\left(P_{O A \_} \max \right)$ is chosen. The difference of the required power is subsequently recovered by re-tuning the gain(s) of the following (downstream) amplifier(s).

\section{OPTICAL POWER CONTROL}

Optical networks are made of optical nodes (ROADMs) interconnected with optical links. In order to achieve maximum network performance, every optical link between two ROADMs is usually designed to support optimum performance independently from other links. With this design method, every link has its own set of optimum span powers and amplifier settings. In this configuration, maximum performance is ensured by setting the optimum power for any new optical channel to $P_{\text {channel, }}$ [14]. This kind of policy does not take into account the fact that channels may require variable reaches; thus some channels may not need the maximum performance (e.g., the channel with the shortest path). As a result some power transmission margins are wasted.

The channel performance and its optical power are tightly linked. Reducing the optical power from its optimum value to a lower value reduces the performance and thus adapts the channel to the required reach. This appears as an interesting method to save some power in a Flex-Grid network and to avoid wasting power transmission margin. More precisely, we expect that this power adaptation will allow the use of the saved margin to increase link capacity in terms of channel number.

To perform the power control, we now propose exploiting the performance estimator of equation (5) of the LOGON strategy in [14]. This equation estimates the OSNR (including non-linear effects in the form of non-linear interference) at the receiver side. If the estimated OSNR $\left(O S N R_{e s t}\right)$ is bigger than the required one $\left(O S N R_{\text {req }}\right)$, then we can adapt channel power. The OSNR value of a lightpath made of successive links is the inverse of the sum of the inverse OSNR of each link [14]. Because OSNR is proportional to channel power and LOGON is already the worst case in terms of nonlinear effects (OSNR overestimation supposing full spectrum load), approximatively every $1 \mathrm{~dB}$ of optical power reduction corresponds to $1 \mathrm{~dB}$ of OSNR reduction [15]. Therefore, as a simplified first guess, we assume that the OSNR margin in $\mathrm{dB}\left(O S N R_{\text {margin }}=O S N R_{e s t}-O S N R_{\text {req }}\right)$ corresponds to the amount of power that can be saved for the related optical channel.

Moreover, since amplifiers have a fixed gain (tuned according to the method explained earlier), this OSNR reduction is simply obtained by tuning the power at the transmitter side: a $x \mathrm{~dB}$ of optical power attenuation at the transmitter side corresponds exactly to $x \mathrm{~dB}$ of power attenuation at the receiver side when passing though the set of spans and amplifiers constituting the optical links. The estimation of the power that can be saved is a rough but simple assumption that can be easily integrated into a control plane. Other methods relying on more complex computation or monitoring mechanisms can be proposed.

\section{GMPLS PROTOCOL AND ROUTING ALGORITHM}

\section{A. GMPLS Protocol Modifications}

Due to the lack of space, the routing and signaling process descriptions of OSPF-TE and RSVP-TE, in addition their 
extensions that are using new collected physical parameters are briefly presented. A detailed description will be the topic of the next publication.

At the end of the design step, every optical link has its own set of configurations for its optical amplifiers; gain and power settings allow computing performance estimator OSNR (equation (5) in [14]). Since no previous work in the domain or any of the IETF RFCs have proposed protocol extensions to include power information for OSPF-TE, we propose modifying it to collect new physical information from the optical plane: $P_{\text {channel, } l}, P_{\text {design,l}}$, and $P_{\text {margin }, l}$, link OSNR as defined in the previous section and real time link power $\left(P(t)_{\text {real }, l}\right)$. We assume that the first four parameters are configured for each link and recorded in the neighboring nodes upon link commissioning. Then, they are collected from the optical plane and placed in the OSPF-TE link state database. The fifth parameter $P(t)_{\text {real }, l}$ is the effective aggregate optical power in the link. It depends on the number of optical channels established at a given $t$ moment. It is updated upon each lightpath setup or release based on optical power computation.

The Path and Resv messages used in the signaling protocol RSVP-TE are also modified to take into account the optical power recommended setting for the lightpath. This enables optical nodes to perform power verification tests during the light path setup (in addition to the wavelength availability test that is usually done). A path computation algorithm was developed to compute paths according to the TE link parameters that we added in the OSPF-TE database.

\section{B. Routing Algorithm}

To find an available and feasible lightpath that satisfies every connection request, we propose the path computation algorithm shown in Fig. 2 and detailed here. For every connection request (i.e., lightpath) between a pair of sources and destination nodes of $T$ Gbit/s rate, it calculates the shortest path using the Dijkstra algorithm. Then, it tries to find a group of $S$ available slots of $12.5 \mathrm{GHz}$ that satisfy the $T$ demand ( $S$ slots are calculated with respect to minimum spectrum occupation, supposing one and the same modulation format for all demands), which are continuous and contiguous using the First-Fit algorithm. The demand is blocked when no available slots are found to satisfy the connection request.

Once this set of free successive optical slots over the path is found, three other tests are performed physical feasibility test, power adaptation (PA), power verification (PV). The physical feasibility test checks whether $O S N R_{\text {est }}$ is above $O S N R_{r e q}$. If the path is physically feasible, then $O S N R_{\text {margin }}$, which is the difference between $O S N R_{\text {est }}$ and $O S N R_{r e q}$, is computed. If $O S N R_{\text {margin }}$ exists, then a channel power adaptation can be made to adapt the optical channel to minimum performances $\left(O S N R_{r e q}\right)$. In this case, the channel power reduction is equal to the $O S N R_{\text {margin }}$ value, and the target optical power for the channel is $P_{\text {channel }}^{\text {adapted }}$ $=P_{\text {channel }}-O S N R_{\text {margin }}$.

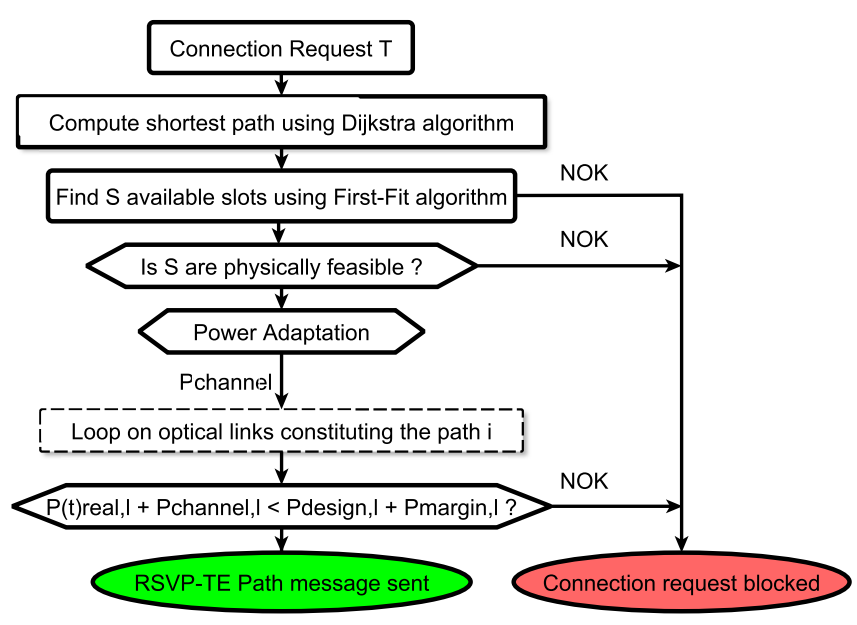

Fig. 2. Path Computation Algorithm.

Regardless of the adapted channel power value, a last power verification test is performed to ensure that this channel, if added, will not cause any saturation problems over the links constituting the optical path. This test consists of comparing the value of the link aggregate power $P(t)_{\text {real, },}$ when adding the new channel $\left(+P_{\text {channel }, l}^{\text {adapted }}\right)$ with the maximum allowed power $\left(P_{\text {design }, l}+P_{\text {margin }, l}\right)$ over every $l$ link constituting the path. These values are made available at each node due to the OSPF-TE link state distribution process. Once these tests are done at the ingress node, the signaling is triggered on the chosen path (i.e., a RSVP-TE Path message is sent downstream in order to set up the optical channel). If any of these tests fails, the connection request is rejected.

Lastly, at each hop, during the signaling process, the aggregate power using the recommended channel power setting is checked in order to verify that it does not exceed the $P_{\max , l}$ of each crossed link. Indeed, if the requests are very frequent, some signaling process may simultaneously compete for the same optical resources in terms of optical power (race condition) and the signaling should avoid any overprovisioning due to the not-yet-updated link database.

\section{Simulation SCENARIO AND RESUltS}

\section{A. Simulation Setup and Scenarios}

In order to test and evaluate the potential gain of our proposed power control, we developed a distributed GMPLSbased network simulator over OMNET++. It simulates OSPFTE and RSVP-TE protocol messages and mechanisms.

We assume that the same initial link design is performed for eighty $100 \mathrm{Gbit} / \mathrm{s}$ QPSK channels over a $50 \mathrm{GHz}$ grid (80*50 $\mathrm{GHz}=4 \mathrm{THz}$ per link) for all scenarios. However, the full usable bandwidth is set to $4.8 \mathrm{THz}$ (optical amplifiers usable bandwidth).

Simulations are performed over the 32 optical nodes and 42 optical links of the European backbone network shown in Fig. 3. Single mode fiber spans are used (chromatic dispersion $=17$ ps. $\mathrm{nm}^{-1} . \mathrm{km}^{-1}$, fiber attenuation $=0.22 \mathrm{~dB} / \mathrm{km}$, non-linearity coefficient $\left.=1 W^{-1} \cdot \mathrm{km}^{-1}\right)$. 


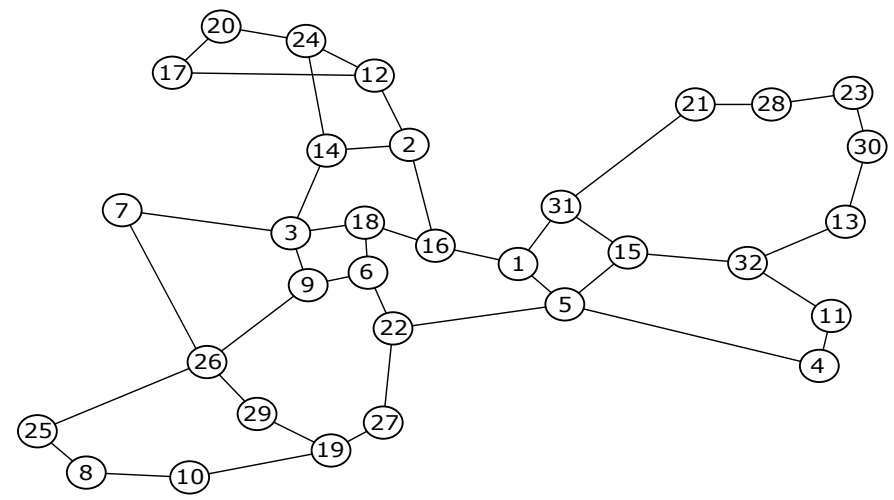

Fig. 3. European Backbone Network Topology.

Links are designed using the three amplifier types presented in Tab. I, and assuming non identical span lengths that are randomly drawn according to a realistic distribution. Filtering penalties induced by transit across one optical node are 0.05 $\mathrm{dB}$ for the $50 \mathrm{GHz}$ channel spacing and $0.64 \mathrm{~dB}$ for the 37.5 $\mathrm{GHz}$ [16]. Tab. I shows the amplifier portfolio comprising several variable gain dual-stage amplifiers without mid-stage access with fixed parameters $\left(P_{O A \_\max }, G_{O A_{-} \max }, F_{1}, F_{2}\right.$, $D)$.

TABLE I

AMPLIFIER MODELS

\begin{tabular}{|c|c|c|c|c|c|}
\hline Type & $P_{O_{A} \_\max }(\mathrm{dBm})$ & $G_{O A_{-} \max }(\mathrm{dB})$ & $F_{1}(\mathrm{~dB})$ & $F_{2}(\mathrm{~dB})$ & Power ratio: $D(\mathrm{~dB})$ \\
\hline $\mathrm{A} 1$ & 17 & 30 & 5 & 6.5 & 3 \\
\hline $\mathrm{A} 2$ & 19 & 25 & 5.5 & 7 & 5 \\
\hline $\mathrm{A} 3$ & 20 & 23 & 6 & 7.5 & 7 \\
\hline
\end{tabular}

In order to simplify the results analysis, just $100 \mathrm{Gbit} / \mathrm{s}$ optical channels are established in all scenarios. The minimum accepted OSNR at the receiver side, using $0.1 \mathrm{~nm}$ noise reference bandwidth, including operational margins, is set to $15 \mathrm{~dB}$ for $100 \mathrm{Gbit} / \mathrm{s}$ QPSK modulation format with coherent detection and soft decision forward error correction (FEC), whatever the channel bandwidth (three or four slots of 12.5 $\mathrm{GHz}$ ). Five scenarios are studied:

- Fixed-Grid (FG): This scenario represents today's core optical networks where no power information is communicated in the control plane. The power control block is not activated in the path computation algorithm or in the protocol. The number of channels that can be set up on a given link is thus limited to 80 , as no other information has been made available, and each channel occupies four contiguous slots.

- Fixed-Grid with power margins (FG4S_PV): In this scenario, the control plane is power aware and thus benefits from the extra power margin of every link $\left(P_{\text {margin }, l}\right)$ to set up channels in the limit of the $4.8 \mathrm{THz}$ bandwidth. The power adaptation block is off, but the power verification is on. Each channel occupies four contiguous slots.

- Fixed-Grid with power control and power margins (FG4S_PA+PV): In this scenario both power adaptation and power verification are allowed. Each individual channel power is tuned to the power satisfying the minimum accepted OSNR value $\left(O S N R_{r e q}\right)$. Each channel occupies four contiguous slots.

- Flex-Grid with power control and power margins (FX3S_PA+PV): This scenario is the same as FG4S_PA+PV but each channel occupies three contiguous slots.

- Flex-Grid with power control and power margins (FX34S_PA+PV): Same as previous scenario, but with the possibility to choose three or four slots of $12.5 \mathrm{GHz}$ for the $100 \mathrm{Gbit} / \mathrm{s}$ channels. The path computation algorithm first assumes three slots of $12.5 \mathrm{GHz}$ for the channel setup. In case the path is not physically feasible (filtering penalty is much higher for three slots than for four slots), the algorithm tries to establish the optical channel using four slots.

Note that in all scenarios, paths that exceed maximum reach (i.e., with OSNR below OSNRreq) are rejected, and we have not implemented regeneration (left for further work).

Fifty simulation runs (with different random seed numbers) were performed for each of the five scenarios with an incremental channel setup (no channel is released). The results depicted in Fig. 4 and 5 are given by averaging the 50 simulation runs with a confidence interval of $95 \%$ (too small to be displayed on the figures). The demand request inter-arrival time in each node follows an exponential law with $\lambda=0.4$. Demand source-destinations are randomly chosen among all source-destination pairs according to a uniform distribution.

\section{B. Simulation Results}

We consider the cumulative blocking probability (CBR) as a first evaluation criterion, which is the ratio of the total number of blocked requests to the total number of generated requests until a time $t$. Figure 4, shows the CBR of the five scenarios as a function of the normalized spectrum occupation of the network, which is the ratio of the total occupied spectrum over all the links of the optical network until a time $t$ to the total spectrum of all the links. Note that on each link, the spectrum occupation corresponds to the number of reserved slots of all channels, each one having three or four slot occupations depending on the scenario.

For all scenarios, CBR at low occupation is not zero because of the rejected demands due to physical feasibility (paths longer than maximum reach). Not surprisingly, since FX3S_PA+PM has a larger filtering penalty, it blocks more demands at low occupation than the other scenarios.

Moreover, FG and FG4S_PV have the same CBR until approximatively $65 \%$ of spectrum occupation. Over $65 \%$ occupation, the CBR of FG4S_PV is smaller because the network benefits from power awareness; it can accept more than 80 channels relying on the remaining power margins over the links. Further, FG4S_PA+PV has a smaller CBR than FG and FG4S_PV because it can not only benefit from the power margin, but it can also generate some power reduction with channel power adaptation. The CBR of FG4S_PA+PV stays below the CBR of FG and FG4S_PV starting from approximatively $26 \%$ of spectrum occupation. This means that 
even at low load, the power reduction enabled by the proposed power control mechanism can be useful.

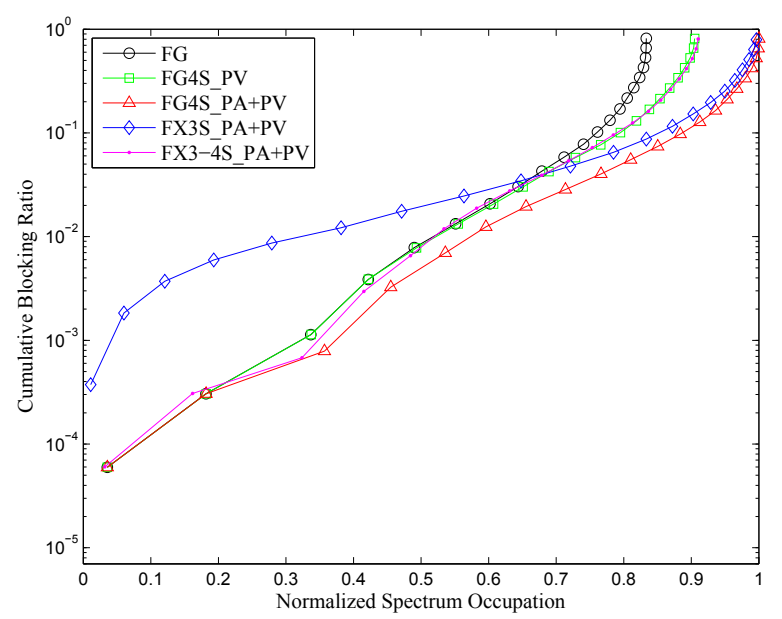

Fig. 4. Cumulative Blocking Ratio vs. Normalized Spectrum Occupation.

We also noticed that blocking for FG4S_PA+PV is only due to physical feasibility and bandwidth availability reasons even at high load, and upon closer investigation of the optical power levels, we noticed that this scenario is not limited by the optical power availability. As explained earlier, the FX3S_PA+PV scenario has bigger CBR at a low occupation ratio because it uses only $37.5 \mathrm{GHz}$ spacing for establishing the $100 \mathrm{Gbit} / \mathrm{s}$ channels; the filtering penalty $(0.64 \mathrm{~dB})$ then reduces the number of feasible paths in the whole network. However, when network load increases, the FX3S_PA+PV CBR is lower than the CBR of FG and FG4S_PV CBR. This is explained first because with three slots per channel, the network can accept more channels than with four slots, and second because the power required for these additional channels has been made available by the control process (PA $+\mathrm{PV})$.

This analysis is confirmed with the FX3-4S_PA+PV scenario. It has a CBR smaller than FX3S_PA+PV for spectrum occupation lower than $65 \%$ because paths rejected due to their non-physical feasibility with $37.5 \mathrm{GHz}$ are established here with $50 \mathrm{GHz}$. Nonetheless, this is paid with lower spectrum efficiency; the spectrum fragmentation caused by the mixing of $37.5 \mathrm{GHz}$ and $50 \mathrm{GHz}$ channels (no spectrum fragmentation aware spectrum assignment) prevents using the whole spectrum bandwidth unlike FG4S_PA+PV and FX3S_PA+PV. This is also confirmed in Fig. 5.

It is important to note that the spectrum efficiency of the FX3S_PA+PV is slightly smaller than FG4S_PA+PV since some links still have spectrum resources but their power resources are completely used at high loads. This is because setting up only three slot channels not only increases the number of channels but also decreases the potential for power saving over links: power adaptation performs less power margins because of the high filtering penalty $(0.64 \mathrm{~dB})$.
We notice that with this network design the amount of $P_{\text {margin,l }}$ of the links is too small to satisfy more than 80 channels (link power margins represent approximately $2.5 \%$ of the available power over the network). In this situation, the power adaptation process is able to save enough power to cancel the blocking for power reasons; scenarios using PA FG4S_PA+PV, FX3S_PA+PV, and FX3-4S_PA+PV have approximately $52 \%, 25 \%$, and $35 \%$, respectively, of remaining power over the entire network (sum of the remaining power over network links).

Figure 5 shows the network capacity (amount of 100 Gbit/s demand accepted and established) as a function of the normalized spectrum occupancy. Note that a four-slot 100 Gbit/s demand going through three optical links (three hop path) for example, will count as $100 \mathrm{Gbit} / \mathrm{s}$ on the $\mathrm{y}$-axis and $3 * 4$ slots $(3 * 50 \mathrm{GHz})$ on the $\mathrm{x}$-axis. This explains why the FG4S_PV and FG4S_PA+PV curve is below the FG one, in addition to the fact that accepted demands in FG4S_PV and FG4S_PA+PV have longer reaches (number of hops) at high load where power blocking appear in FG. This explanation also holds for FG4S_PV versus the FG curve, and it is particularly visible in the FX3S_PA+PV scenario, which has much shorter paths on average than all the other scenarios.

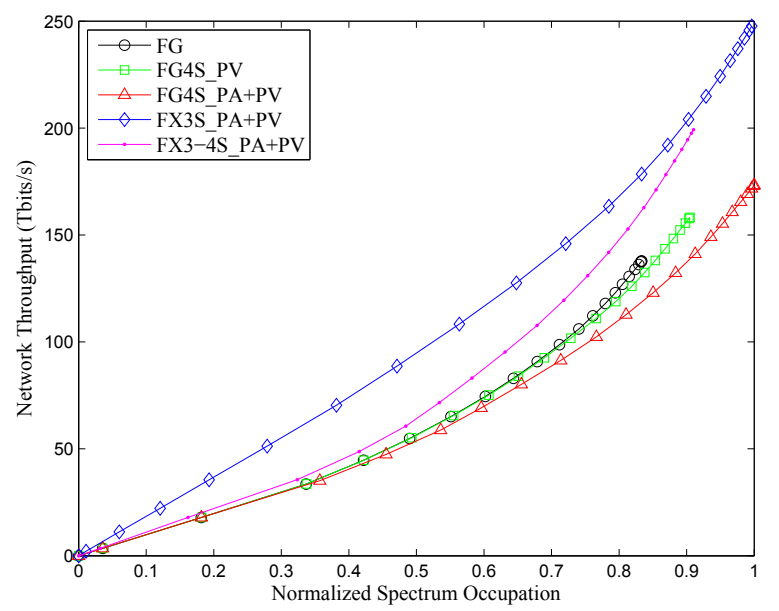

Fig. 5. Network Throughput vs. Normalized Spectrum Occupation.

The FG, FG4S_PV, and FG4S_PA+PV reach 137.8 Tbit/s, 158.2 Tbit/s, and $173.3 \mathrm{Tbit} / \mathrm{s}$, respectively, of carried traffic. Therefore, the power control has increased the capacity of the Fixed-Grid network of approximately 25\%. As expected, the power control coupled with the use of the Flex-Grid with FX3S_PA+PV greatly increases the network capacity to 248 Tbit/s. This represents $80 \%$ of the capacity increase compared to FG (i.e., accounting for the $0.8 \mathrm{THz}$ more total spectrum resource) and $45 \%$ when compared to FG4S_PA+PV. We also note that the Flex-Grid scenario mixing three and four slot channels has a larger capacity than FG4S_PA+PV despite the fact that it can occupy less spectrum. These results mean that power control with power adaptation is an efficient mechanism 
to benefit from the link total spectrum bandwidth, without the need to re-design the existing optical network.

\section{CONCLUSION}

In this paper, we have addressed the optical amplifier power limitation issue that an operator network planner will face when migrating networks from Fixed-Grid to Flex-Grid networks. Using a developed link design method that we detail in this paper, we have proposed a channel power control process with power information distribution, power verification, and power adaptation. We also describe a path computation algorithm that includes power control and show how the whole process can be integrated into a distributed GMPLS-based control plane. We suggest several modifications for the existing OSPF-TE and RSVP-TE protocols to include power information.

It is important to emphasize that our power control process is completely independent from link design, OSNR estimator, or control plane protocol. Any other link design method associated with any OSNR estimator could be used to perform the power control.

Simulation results revealed that the power control process is an efficient way to benefit from Flex-Grid capacity promises while maintaining the use of legacy amplifiers without the need to re-design any links in the network. Future work will include other network topologies, in addition to a detailed description of control plane protocol extensions and mechanisms for OSPF-TE and RSVP-TE protocols to be able to apply the power control over a distributed-GMPLS based network. The power control process will also be evaluated in the dynamic case where connections are established and released.

\section{ACKNOWLEDGMENTS}

This work was partly supported by the DGCIS, in the frame of the CELTIC-Plus project SASER-SIEGFRIED.

\section{REFERENCES}

[1] O. Gerstel, M. Jinno, A. Lord, and S. B. Yoo, "Elastic optical networking: A new dawn for the optical layer?" Communications Magazine, IEEE, vol. 50, no. 2, pp. s12-s20, 2012.
[2] P. Wright, A. Lord, and L. Velasco, "The network capacity benefits of Flexgrid," in Optical Network Design and Modeling (ONDM), 2013 17th International Conference on. IEEE, 2013, pp. 7-12.

[3] "ITU-T Recommendation G.694.1, Spectral grids for WDM applications: DWDM frequency grid."

[4] P. Poggiolini, "The GN Model of Non-Linear Propagation in Uncompensated Coherent Optical Systems," Journal of Lightwave Technology, vol. 30, no. 24, pp. 3857-3879, Dec. 2012.

[5] F. Vacondio, C. Simonneau, L. Lorcy, J.-C. Antona, A. Bononi, and S. Bigo, "Experimental characterization of Gaussian-distributed nonlinear distortions," in European Conference on Optical Communications. Optical Society of America, 2011, pp. We-7.

[6] A. Carena, V. Curri, G. Bosco, P. Poggiolini, and F. Forghieri, "Modeling of the Impact of Nonlinear Propagation Effects in Uncompensated Optical Coherent Transmission Links," Journal of Lightwave Technology, vol. 30, no. 10, pp. 1524-1539, May 2012.

[7] G. Bosco, A. Carena, R. Cigliutti, V. Curri, P. Poggiolini, and F. Forghieri, "Performance prediction for WDM PM-QPSK transmission over uncompensated links," in Optical Fiber Communication Conference. Optical Society of America, 2011.

[8] D. J. Ives and S. J. Savory, "Transmitter optimized optical networks," in National Fiber Optic Engineers Conference. Optical Society of America, 2013, pp. JW2A-64.

[9] D. J. Ives, P. Bayvel, and S. J. Savory, "Adapting Transmitter Power and Modulation Format to Improve Optical Network Performance Utilizing the Gaussian Noise Model of Nonlinear Impairments," Journal of Lightwave Technology, vol. 32, no. 21, pp. 4087-4096, Nov 2014.

[10] J. Auge, "Can we use flexible transponders to reduce margins?" in $O p$ tical Fiber Communication Conference/National Fiber Optic Engineers Conference, 2013.

[11] A. Mitra, S. Kar, and A. Lord, "Effect of frequency granularity and Link Margin at $100 \mathrm{~g}$ and beyond Flexgrid Optical Networks," in Communications (NCC), 2014 Twentieth National Conference on. IEEE, 2014, pp. 1-5.

[12] A. Mitra, A. Lord, S. Kar, and P. Wright, "Effect of link margin and frequency granularity on the performance of a flexgrid optical network," Optics express, vol. 22, no. 1, pp. 41-46, 2014.

[13] D. Amar, M. Kanj, J.-L. Auge, N. Brochier, E. Le Rouzic, C. Lepers, and B. Cousin, "On legacy amplifier limitation in flexgrid optical networks," in International Conference on Photonics in Switching, 2015 (Accepted).

[14] P. Poggiolini, G. Bosco, A. Carena, R. Cigliutti, V. Curri, F. Forghieri, R. Pastorelli, and S. Piciaccia, "The LOGON strategy for lowcomplexity control plane implementation in new-generation flexible networks," in Optical Fiber Communication Conference. Optical Society of America, 2013, pp. OW1H-3.

[15] F. Vacondio, O. Rival, C. Simonneau, E. Grellier, A. Bononi, L. Lorcy, J.-C. Antona, and S. Bigo, "On nonlinear distortions of highly dispersive optical coherent systems," Optics Express, vol. 20, no. 2, pp. 1022-1032, 2012.

[16] D. Amar, E. Le Rouzic, N. Brochier, J.-L. Auge, C. Lepers, N. Perrot, and S. Fazel, "How problematic is Spectrum Fragmentation in operator's Gridless network?" in Optical Network Design and Modeling, 2014 International Conference on. IEEE, 2014, pp. 67-72. 